JLL 6 (1)(2019)

\title{
Le Registre Argotique au Twitter Français dans La Perspective Sociolinguistique
}

\section{Eli Prastiwi ${ }^{\bowtie}$ Bernardus Wahyudi Joko Santoso}

Département de la Langue et la Littérature Étrangère, Faculté des Langues et des Arts, Universitas Negeri Semarang, Indonesia

\section{Info d'article Extrait}

Histoire de l'article:

Reçu fevriér 2019

Accepté avril 2019

Publié mai 2019

\section{Mots-clés:}

Régistre argotique, Twitter,

Sens Dénotatif, Sens

Connottaif, Fonction de

langage
Les objectifs de cette recherche sont de décrire la forme du registre argotique, le sens dénotatif, le sens connotatif, et les fonctions de langage dans le twitter français. C'est une recherche utilisé l'approche théorétique de la sociolinguistique et utilisé l'approche méthodologique descriptive analytique qualitative. Les données de cette recherche sont les paroles des usageurs twitter français. Elles ont eu pris de www.twitter.com d'août à septembre 2017. La méthode de recueillir des données dans cette recherche est la méthode simak (la méthode d'épargne) avec la technique de basse sadap (la technique de taraudage de langue) et les techniques avancées : la technique SBLC (le chercheur ne participe pas à la conversation réelle) et la technique catat (le chercheur a obtenu les données de prise de notes dans la carte de données) ou la classification des données étant présentée dans des tableaux. J'ai appliqué la méthode agih (la méthode de distribution) en utilisant la technique Bagi Unsur Langsung (le chercheur a divisé les éléments de données directement) et les techniques avancées : la technique ganti (la technique de substitution) et la technique perluas (la technique d'extension) pour analyser les formes du registe argotique et les fonctions de langue, $\mathrm{j}$ 'ai utilisé aussi l'analyse componentielle du sens par Nida pour analyser le sens dénotatif et le sens connotatif. Les résultats de cette recherche montrée qu'il y a 32 mots argotiques et 1 syntagme argotique. Chaque mot argot a le sens dénotatif et le sens connotatif. Il y a 5 fonctions de langue utilisées dans les paroles. Les 5 fonctions utilisées sont la fonction personnelle (8 données), interactive (4 données), informative ( 7 données), imaginative (1 donnée), personnelle + regulative (1 donnée), et personnelle + informative ( 3 données). Selon les résultats d'analyse, l'utilisation du registre argotique est nécessaire pour que pas tous les usageurs de twitter comprennent ce qui est locuteur était dire, sauf les usageurs prévus. Cela correspond à la fonction du registre argotique qui a la fonction comme une langue secrèt pour un group particulier. La fonction de langue dominante utilisé est la fonction personnelle et informative. Il montre que les locuteurs ont tendance à exprimer des sentiments, la conscience de soi et partage des informations directement. Cela correspond à la fonction de twitter qui est médias sociaux à partager. 


\section{INTRODUCTION}

La sociolingustique est une etude de notre vie quotidienne, comment la langue utilise dans nos conversations occasionnelles, les médias nous avons exposés, et la présence de normes sociales, les politiques et les lois qui concernent la langue (Wardhaugh 2015 :1).

Dans le français, il y a plusieurs registres de la langue c'est comme le registre courant, registre soutenu, le registre famillier, le registre populaire, etc. La branche du registre populaire est le registre argotique.

Bonnard, cité par Colin (2002: 865) a dit que argot attesté d'abord au sens de « corporation de voleurs » (1628), le mot argot a designé depuis soit un vocabulaire secret, réservé à un groupe d'initiés coupeurs de bourses, travailleurs, étudiants, soit un ensemble de termes insolites tenus pour vulgaires, et jugés expréssifs par cela même et/ou par les qualités stylistiques qui ont fait leur fortune dans le peuple ou la pègre.

$\mathrm{La}$ variation linguistique peut utiliser dans la communication verbale ou non verbale. $\mathrm{La}$ communication verbale est la forme de communication du locuteur à l'interlocuteur dans la forme orale ou ecrit, et la communication non verbale est la forme communication du locuteur à l'interlocuteur dans la forme gestuele, l'expréssion du visage, et le contact des yeux (Chaer 2004 :20).

La communication verbale peut été trouvé dans la conversation quotidienne : dans la société, à la radio, aux les médias sociaux, à la télévision, etc. Aux médias sociaux, on peut utiliser le facebook, instagram, snapchat, twitter, etc. Il y a plusieurs registres de la langue aux médias sociaux. Un des quelques registres de langue est le régistre argotique. Dans cette recherche, j'ai choisi le régistre argotique dans le statut de twitter français car j'ai suivi les utilisateurs de twitter français, je voudrais enrichir les vocabulaires françaises notamment les vocabulaires dans le registre argotique, et les étudiants français en étrangères ont la difficulté de comprendre ce régistre.

Cette recherche a recherché les registres argotiques, le sens dénotatif, le sens connotatif, et la fonction de langage qui ont utilisé dans le twitter fraçais. Elle a l'objectif, ce sont de décrire les registres argotiques, le sens dénotatif, le sens connotatif, et les fonctions de langage dans le twitter français.

\section{THEORIE}

La recherche du régistre ou la variation de langue dans le français a été examinée plusieurs fois, c'était comme celle de Trollvin dans le titre Une Etude des Différences Types d'Argot et de Leurs Effects Provocateurs. Elle a etudié les différences entre la langue argotique et les effects provocatifs qui ont infligé par la langue argotique dans la chanson française moderne.

Pour soutenir cette recherche, j'ai besoin de quelques théories par des experts comme des références scientifiques. Ce sont les théories utilisées dans cette recherche.

\section{Sociolinguistique}

Fishman cité par Chaer (2004:3) dit que la sociolinguistique recourt au terme de variété au lieu de langue sans en donner une défiition concise.

Saussure cité par Chaer (2004:2) dit que la langue n'est pas une fonction du sujet parlant, elle est le produt que l'individu enregistre passivement, elle est la partie sociale du langage, elle est le produit que l'individu passivement. Alors, la sociolinguistique est la rélation entre la langue et la société.

La sociolinguistique est une discipline qui, comme son nom l'indique, étudie à la fois l'usage de la langue dans la société et le phénomène structurel de la langue. Le premier renvoie à tous les facteurs qui déterminent l'emploi de la langue au sein de la société et l'étude structurelle de la langue 
se base sur les aspects formels du discours, comme le style, les phénomènes, les variétés, etc (Haque $2006: 23)$.

\section{Fonctions de Langage}

En général, la langue sert de moyen de communication ou de moyen d'interaction. Les experts ont classifié les fonctions de langue d'après eux même. Ce sont commes les fonctions de langue d'après Malinowski, Karl Bühler, Desmond Morris, James Britton, et Halliday. Dans cette recherche, j'ai utilisé les fonctions de langue d'après Halliday.

Halliday a classifié les fonctions de langue dans sept fonctions, ce sont la fonction instrumentale, cette fonction exée sur la satisfaction de besoins divers l'obtentin de biens et de services. La deuxième fonction est la fonction régulatrice, cette fonction utilisée pour contrôler le comportement d'autrui. La troisième fonction est la fonction interactive, cette fonction utilisée pour entrer en contact avec autrui dans l'attente d'une réponse. La quatrième fonction est la fonction personnelle, cette fonction permet à son auteur ou auteure d'exprimer son caractère unique, sa conscience de soi, ses goûts, ses sentiments et ses opinins. La cinquième fonction est la fonction imaginative, cette fonction sert à créer un environnement de son cru, entièrement ictif, à explorer le langage, à se construire un imaginaire. La sixième fonction est la fonction heuristique, cette fonction a utilisé pour recouvre l'ensemble des types de questions qu'on apprend à se poser dans la recherche du savoir. La septième fonction est la fonction informative, cette fonction a utilisé pour permettre la transmission ou de se donner des informations (Djojosuroto 2006 : 42-44).

\section{Concept du Régistre}

Auger cité par Moureau (1997) a dit que le terme registre a été proposé par Reid dans les années 1950 pour désigner une variété linguistique appropriée à une situation particulière. De même, pour Ferguson, l'étiquette renvoie à sous-système linguistique caractérisé par un certain nombre de constructions spécifiques et réservé à des situations circonscrites.

Les registres de la parole sont les utilisations que chaque sujet parlant fait des niveaux de langue exitant dans l'usage social d'une langue (Dubois 2001 : 406). Pour comprendre le concept du registre, regardez l'exemple suivant :

(1) Il était une fois, un homme très pauvre vivait au cœur d'une forêt.

Dans cette exemple, on peut dire que on est en train de lire l'histoire traditionnelle, parce qu'il y a le registre qui marque cette phrase, c'est il était une fois. Il est le registre courant ou le registre qui a utilisé dans le français standard.

Un de quelques registres français est le registre argotique. Pour comprendre la théorie du registre argotique, regardez-le ci-dessous.

\section{Registre Argotique}

Vendys citée par Carillo (1987 : 737) dit que dans le français, il y a le registre argotique. Il est la branche du registre populaire. Ce registre a utilisé comme l'objet dans cette recherche.

Geiger cité par Colin (2002:875-876) dit que pour les francophones, argot évoque avant tout l'argot parisien de la fin du XIX ${ }^{\mathrm{e}}$ siècle et du début du XX $\mathrm{XX}^{\mathrm{e}}$, celui de Jehan Rictus et de Bruant. Avec un brin de goût subversif, comme si l'on sentait bien que ce n'est pas sans raisons que nombre d'argotiers disent que c'est un art de vivre, une façon d'être qui engendre un habitus

Utilisation du registre argotique a developpé dans le banlieu, dans cette place il y a des immigrants, des tueurs, et des gangsters. L'argot demeure, en première ligne, le parler des truands, grands ou petit. Jour après jour, le registre argotique a utilisé par des adolescences, des étudiants, des jeunes gens, et les autres communautés. Ils l'utilisent dans la parole non formelle. 


\section{Sens Dénotatif et Connotatif}

Keraf (2009:27-29) dit que la forme des mots communément parlé dans la grammaire. Il y a les sens des mots, ce sont le sens dénotatif et le sens connotatif. Le sens dénotatif est le sens du mot qui livre directement et tout le monde d'accord avec le mot ou phrase qui a dit par le locuteur. Le sens connotatif est le sens qui a la valeur émotionnelle, ce sens a les sentiments d'accord et pas d'accord, heureux et pas heureux du locuteur à l'interlocuteur.

\section{Twitter}

Le twitter est un réseau social de microblogage géré par l'entreprise Twitter Inc. Il permet à un utilisateur d'envoyer gratuitement de brefs messages, appelés tweets, sur internet, par messagerie instantanée ou par SMS. Ces messages sont limités à 140 caractères. (Fitton 2009 :1)

Les usageurs de twitter ont ses caractéristiques. Domenget (2017:14) dit que les usageurs de twitter ont quatre caractéristiques, ce sont twitto, consommateur, abonnée, et expert. Le twitto est la figure de l'usager de twitter médiatisée les médias de masse auprès du grand public, lequel reste souvent aujourd'hui encore non usager de dispositif. Le consommateur relève du principe de contribution, correspondant à la vision d'un usager actif. L'abonnée relève du principe de représentativité et est issue de systèmes de mesure d'audience de recommandation. L'expert relève d'un participe d'une opinion au sein d'un collectif d'usager " parce qu'on lui reconnaît une certain autorité ».

\section{METHODOLOGIE DE LA RECHERCHE}

L'approche utilisée dans cette recherche est l'approche théorétique, c'est l'approche sociolinguistique et l'approche méthodologique, c'est l'approche descriptive analytique qualitative sous la forme d'étude de cas. Elle a recherché le cas ou le phénomène dans la communauté pour étudier sa formation, sa circonstance et ses interactions arrivées. Utilisation de cette approche a pour but de décrire le registre argotique, le sens dénotatif et le sens connotatif, et de décrire les fonctions de langage au twitter français. La méthode de recueillir des données dans cette recherche est méthode simak (la méthode d'épargne) avec la technique de basse sadap (la technique de taraudage de langue) et les techniques avancées : la technique SBLC (le chercheur ne participe pas à la conversation réelle) et la technique catat (le chercheur a obtenu les données de prise de notes dans la carte de données) ou la classification des données étant présentée dans des tableaux. J'ai appliqué la méthode agih (la méthode de distribution) en utilisant la technique Bagi Unsur Langsung (le chercheur a divisé les éléments de données directement) et les techniques avancées : la technique ganti (la technique de substitution) et la technique perluas (la technique d'extension) pour analyser les formes du registe argotique et les fonctions de langue, j'ai utilisé aussi l'analyse componentielle du sens par Nida pour analyser le sens dénotatif et le sens connotatif.

\section{ANALYSE}

Dans cette recherche j'ai analysé le registre argotique, le sens dénotatif, le sens connotatif et la fonction de langue dans la parole du twitter. Elle a utilisé 14 données qui a 25 paroles, ces paroles ont 31 mots argot et 1 phrase argot, et il y a 5 fonction de langue utilisée. Les résultats de la recherche seront présentés dans les tableaux suivant.

\section{Tableau 4.1}

Le Tableau de Résultat du Registre Argotique, du Sens Dénotatifet du Sens Connotatif

\begin{tabular}{|l|l|l|l|l|}
\hline $\mathrm{N}^{\circ}$ & Forme Argotique & Sens Dénotatif & $\begin{array}{l}\text { Sens } \\
\text { Connotatif }\end{array}$ & $\begin{array}{l}\text { Total de } \\
\text { Résultats }\end{array}$ \\
\hline
\end{tabular}


Eli Prastiwi, etc. / Journal of Lingua Littératia 6 (1) (2019)

\begin{tabular}{|c|c|c|c|c|c|}
\hline & Mot & Syntagme & & & \\
\hline 1 & $\operatorname{mec}(n . m)$ & & $\begin{array}{l}\text { individu de sexe } \\
\text { masculine }\end{array}$ & un agresseur & 6 \\
\hline 2 & $\operatorname{meuf}(n . f)$ & & $\begin{array}{l}\text { individu de sexe } \\
\text { feminine }\end{array}$ & $\begin{array}{l}\text { femme } \\
\text { lubrique }\end{array}$ & 5 \\
\hline 3 & putain (n.f) & & Prostituée & $\begin{array}{l}\text { femme de vie } \\
\text { dissolue }\end{array}$ & 3 \\
\hline 4 & potes (n.m) & & $\begin{array}{l}\text { copain (e), ami } \\
\text { (e) }\end{array}$ & $\begin{array}{l}\text { amitie entre } \\
\text { l'homme }\end{array}$ & 3 \\
\hline 5 & $\operatorname{merde}(n \cdot f)$ & & $\begin{array}{ll}\text { excrement } & \text { de } \\
\text { personne } & \text { ou } \\
\text { d'animal } & \end{array}$ & Calomnie & 2 \\
\hline 6 & taff (n.m) & & travail à faire & Peur & 2 \\
\hline 7 & baltringue (n.m) & & $\begin{array}{lr}\text { se dit d'un } \\
\text { individu } & \\
\text { étrangère } & \text { au } \\
\text { milieu } & \end{array}$ & $\begin{array}{l}\text { personne qui } \\
\text { n'a pas de } \\
\text { parole }\end{array}$ & 1 \\
\hline 8 & bordel (n.m) & & $\begin{array}{l}\text { établissement } \\
\text { où se pratique a } \\
\text { prostitution }\end{array}$ & $\begin{array}{l}\text { situation } \\
\text { chaotique }\end{array}$ & 1 \\
\hline 9 & bouclent (v) & & Fermer & Fermer & 1 \\
\hline 10 & & $\begin{array}{l}\text { Faire du } \\
\text { Boudin (s.v) }\end{array}$ & $\begin{array}{l}\text { témoigner par } \\
\text { une certaine } \\
\text { expression du } \\
\text { visage }\end{array}$ & $\begin{array}{l}\text { faire comme } \\
\text { l'enfant }\end{array}$ & 1 \\
\hline 11 & boulot (n.m) & & travaille & $\begin{array}{l}\text { avoir une } \\
\text { résistance }\end{array}$ & 1 \\
\hline 12 & cool (adj.) & & Calme & $\begin{array}{l}\text { se sentir } \\
\text { innosents }\end{array}$ & 1 \\
\hline 13 & fan (n.m) & & $\begin{array}{l}\text { admiratrice } \\
\text { enthousiaste } \\
\text { d'une personne } \\
\text { ou d'une chose }\end{array}$ & $\begin{array}{l}\text { personne qui } \\
\text { adhère } \\
\text { totalement }\end{array}$ & 1 \\
\hline 14 & gueule (n.f) & & Bouche & $\begin{array}{l}\text { bouche chez } \\
\text { les animaux }\end{array}$ & 1 \\
\hline 15 & nique (n.f) & & $\begin{array}{l}\text { posséder } \\
\text { sexuellement }\end{array}$ & Coït & 1 \\
\hline 16 & pute (n.f) & & prostituée & $\begin{array}{l}\text { femme de vie } \\
\text { dissolue }\end{array}$ & 1 \\
\hline 17 & titis (n.m) & & $\begin{array}{l}\text { jeune } \\
\text { parisienne } \\
\text { gouailler }\end{array}$ & $\begin{array}{l}\text { garçon } \\
\text { dégourdi }\end{array}$ & 1 \\
\hline 18 & $\operatorname{trucs}(n . m)$ & & quelque chose & $\begin{array}{l}\text { activité } \\
\text { délictueuse }\end{array}$ & 1 \\
\hline \multicolumn{4}{|l|}{ Total } & \multicolumn{2}{|c|}{$\begin{array}{l}32 \text { mots argotiques et } 1 \\
\text { syntagme verbal argotique }\end{array}$} \\
\hline
\end{tabular}


Tableau 4.2

Le Tableau de Résultats de la Fonction de Langage

\begin{tabular}{|l|l|l|l|}
\hline $\mathrm{N}^{\circ}$ & Fonction de Langage & Dans le Data & Total de Résulats \\
\hline 1 & Personnelle & $1,2,10,12,13,16,17$, et 24 & 8 paroles \\
\hline 2 & Informative & $5,9,14,15,20,21$, et 23 & 7 paroles \\
\hline 3 & Interactive & $4,6,8$, et 25 & 4 paroles \\
\hline 4 & Regulative & 3 & 1 parole \\
\hline 5 & Imajinative & 11 & 1 parole \\
\hline 6 & personelle + regulative & 7 & 1 parole \\
\hline 7 & personelle + informative & 18,19, et 22 & 3 paroles \\
\hline Total & & 25 paroles \\
\hline
\end{tabular}

Ensuite, j'ai relevé un exemple pour chaque type d'analyse. Voilà les exemples d'analyse des données.

\section{Registre Argotique, Sens Dénotatif, et Sens Connotatif}

(1) La parole de Ln (@MarionSeleanor)

Et le bus qu'à pas l'air de vouloir passer c'est une blague, quelle matinée de merde qui s'annonce. (twitter.com)

Dans cette parole, il y a le registre argotique 'merde' (n.f). Le mot 'merde' a le sens dénotatif 'l'excrement d'humain ou des animaux' et a le sens connotatif 'infortuné'.

Pour vérifier que le mot 'merde' est le registre argotique, on peut utiliser la technique ganti. La technique ganti utilisée pour savoir la similarité de la chategorie du mot. Avec cette technique, la parole (1) devient la parole (1a) ci-dessous.

(1a) Et le bus qu'à pas l'air de vouloir passer c'est une blague, quelle matinée d'excrément qui s'annonce.

Après vérifiée avec cette technique, on peut dire que le mot 'merde' est le registre argotique de registre courant 'excrément'. Les deux mots peuvent se remplacer car ils ont la même catégorie, c'est le nom.

\section{La Fonction de Langage}

\section{La Fonction Personnelle}

(26) La parole de Ln (@MarionSeleanor)

Et le bus qu'à pas l'air de vouloir passer c'est une blague, quelle matinée de merde qui s'annonce. (twitter.com) né.

La parole (26) est la fonction personnelle, car elle a soutenu par le registre argotique 'merde' qui a le sens dénotatif connotatif 'infortuné'. Cette fonction a vue quand $\mathrm{Ln}$ (MarionSeleanor) voulait utiliser le bus, mais elle a regardé le bus n'eut pas fonctionné. Alors, elle a utilisé le mot 'merde' pour exprimer sa colère.

Pour vérifier la fonction personnelle au dessus, la technique d'extension peut utiliser. Cette technique est importante pour connaître des niveaux de synonymie. Les niveaux de synonymie ici n'est pas pour connaître la similitude, mais pour connaître la différence. Avec cette technique, la parole (26) devient la parole (26a) ci-dessous.

(26a) Il faut être capable de se saisir de la noirceur, de la douleur, de la merde, pour en faire un plaisir. 
La parole (26a) est la fonction regulative car cette parole a le sens que le locuteur voulait l'interlocuteur faire ce qu'il voulait, il demandé aux autres pour combattre la douleur pour faire un plaisir. Avec cette vérification, on peut dire que la ( $1^{\text {ère }}$ donnée) a la fonction personnelle.

\section{La Fonction Interactive}

(38) La parole de Ln (@MarionSeleanor)

Vais je un jour arrêter de lâcher des disquettes à mes potes? (twitter.com) ${ }^{\grave{\mathrm{O}}}$

La parole (38) est la fonction interactive, car elle voulait gagner au contact social dans le twitter qui a montré par le locuteur, c'est Ln (@MarionSeleanor) a donné la question aux usageur de twitter avec son tweet. La parole de cette donnée a montreé un des caractéristiques de la fonction interactive, c'est gagner le contact dans la forme de réponses de communauté de twitter.

La technique d'extension peut utiliser pour vérifier que la parole (38) est la parole qui utilise la fonction interactive. Alors, en utilisant cette technique, la parole (38) devient la parole (38a) cidessous.

(38a) Mes potes sont gentilles.

La parole (38a) a la fonction informative, car le locuteur a donné l'information que ses amis sont gentilles. Avec cette vérification, nous pouvons dire que la (4⿳亠㐅े donnée) a la fonction interactive.

\section{La Fonction Informative}

(42) La parole de manon (@ItsAmazynx)

Nique sa race le bac.(twitter.com)

(5’̀̀ donnée)

La parole (42) a la function informative, car la parole de manon (@ItsAmazynx) voulait donner l'information a l'interlocuteur que l'enfant de la personne dans sa parole est un le bac. Cette parole a la caractéristique de 1 fonction informative, c'est donner l'information à l'interlocuteur.

La technique d'extension peut utiliser pour vérifier que la parole (42) a la fonction informative. Alors, la parole (42) devient la parole (42a) ci-dessous.

(42a) Mais arrête de lui faire la nique!

La parole (42a) a la fonction regulatrice, car le locuteur a donné l'influence à l'interlocuteur de ne pas faire la nique avec la personne qu'il voulait dire dans sa parole. Avec cette vérification, nous pouvons dire que la ( $5^{\grave{e}}$ donnée) a la fonction informative.

\section{La Fonction Imaginative}

(52) La parole de Kreyz (@Kreyz_)

J'veux trouver qui m'aide à boire quand j'suis allongé comme le mec derrière, goal (twitter.com)

La parole (52) a la fonction imaginative, parce que Kreyz (@Kreyz_) a imaginé si'il devient le haut comme l'homme derrière, il va trouver la personne qui lui aide à boire. Cette parole montré la caractéristique de la fonction imaginative, c'est créer un environnement de son cru sous la forme d'imagination / fiction.

La technique d'extension peut utiliser pour vérifier que la parole (52) est la parole qui utilise la fonction imaginative. Alors, en utilisant cette technique, la parole (52) devient la parole (52a) cidessous.

(52a) Le mec de Jeanne est très beau. 
La parole (52a) a la fonction personnelle, parce que le locuteur a exprimé son opinion que le mec de Jeanne est très beau. Avec cette vérification, nous pouvons dire que la (11 ${ }^{\mathrm{e}}$ donnée) a la fonction imaginative.

\section{La Fonction Regulative}

(52) Tuturan dari akun lichou (@liabricot)

Bon les mecs gros thread sur la mondialisation! Soyez attentifs!(twitter.com)

La parole (53) a la function regulative, parce que lichou (@liabricot) a affecté les hommes au twitter a fait attention à la globalisation. Cette parole montre la caractéristique de la fonction regulative, c'est affecter les autres.

La technique d'extension peut utiliser pour vérifier que la parole (53) est la parole qui utilise la fonction regulative. Alors, en utilisant cette technique, la parole (53) devient la parole (53a) ci-dessous.

(53a) C'est mon mec, Pierre.

La parole (53a) a la fonction informative, car le locuteur a donné l'infomation à l'interlocuteur que Pierre est son mec. Avec cette vérification, nous pouvons dire que la (11 ${ }^{\mathrm{e}}$ donnée) a la fonction imaginative.

\section{LA CONCLUSION}

Selon les résultats de l'analyse, l'utilisation du registre argotique dans le twitter français a un but pour que pas tous les twitter usager commprennnent ce qui est locuteur était dire, sauf l'interlocuteur qui a visé de parler. Cela corespond à la fonction du registre argotique qui a la fonction comme une langue secrète pour un group particulier. Les résultats de cette recherche montrée qu'il y a 32 mots argotiques et 1 syntagme verbale argotique. Le sens dénotatif du registre argotique est toujours le même quand la phrase a utilisé le même mot. Le sens connotatif du registre argotique peut être différent ça dépend de la contexte qui a eu utilisé. La fonction de langage dominante utilisé est la fonction personnelle et informative. La fonction personnelle indique que le locuteur voulait exprimer ses sentiments, son effusion de cœur, ses opinions, et ses émotions. La fonction informative indique que le locuteur voulait partager des informations directes à l'interlocuteur. Ces deux choses correspondent à la fonction de twitter qui est médias sociaux à partager. Il y a 5 fonctions de langage utilisées dans les paroles. Les 5 fonctions utilisées sont la fonction personnelle (8 données), interactive (4 données), informative ( 7 données), imaginative (1 donnée), personnelle+regulative (1 donnée), et personnnelle+informative ( 3 données). Il ya 2 fonctions de langage n'utilisées pas dans les paroles, ce sont la fonction heuristique et la fonction instrumentale. 